\title{
ON THE DISTRIBUTION OF PRIMES IN SHORT INTERVALS
}

\author{
P. X. GALLAGHER
}

One of the formulations of the prime number theorem is the statement that the number of primes in an interval $(n, n+h$ ], averaged over $n \leqslant N$, tends to the limit $\lambda$, when $N$ and $h$ tend to infinity in such a way that $h \sim \lambda \log N$, with $\lambda$ a positive constant.

In this note we study the distribution of values of $\pi(n+h)-\pi(n)$, for $n \leqslant N$ and $h \sim \lambda \log N$. We show that, assuming a certain uniform version of the (unproved) prime $r$-tuple conjecture of Hardy and Littlewood [3], the distribution tends to the Poisson distribution with parameter $\lambda$ as $N \rightarrow \infty$. Using a sieve upper bound for the $r$-tuple problem, we also get an unconditional exponential upper bound for the tail of the distribution.

Our method has many features in common with the argument by which Hooley [4] has studied the distribution of values of the differences between consecutive integers prime to $n$, for $n / \phi(n)$ large. An analogous result for primes has been announced by Hooley in [5].

Explicitly, the $r$-tuple conjecture is an asymptotic formula for the number $\pi_{\mathrm{d}}(N)$ of positive integers $n \leqslant N$ for which $n+d_{1}, \ldots, n+d_{r}$ are all prime. Here $d_{1}, \ldots, d_{r}$ are distinct integers. The formula is

$$
\pi_{\mathrm{d}}(N) \sim \mathscr{S}_{\mathrm{d}} \frac{N}{\log ^{r} N} \quad(N \rightarrow \infty),
$$

provided $\mathscr{S}_{\mathrm{d}} \neq 0$, where

$$
\mathscr{S}_{\mathrm{d}}=\prod_{p} \frac{p^{r-1}\left(p-v_{\mathrm{d}}(p)\right)}{(p-1)^{r}},
$$

and where $v_{\mathrm{d}}(p)$ is the number of distinct residue classes mod $p$ occupied by $d_{1}, \ldots, d_{r}$.

Formula (1) is the prime number theorem, for $r=1$. For $r \geqslant 2$, it has not been proved for any $\mathbf{d}$; the source of (1) in these cases is a heuristic application of the circle method, and a summation of the corresponding (multiple) singular series [3]. Lavrik [8] has proved that (1) holds in mean over cubes $1 \leqslant d_{1}, \ldots, d_{r} \leqslant H$, in the range $N / \log ^{c} N \leqslant H \leqslant N$; a similar mean result for the (small) cubes of side $h$ would suffice for our purpose.

THEOREM 1. Denote by $P_{k}(h, N)$ the number of integers $n \leqslant N$ for which the interval $(n, n+h]$ contains exactly $k$ primes. Then

$$
P_{k}(h, N) \sim N \frac{e^{-\lambda} \lambda^{k}}{k !}
$$

for $N \rightarrow \infty, h \sim \lambda \log N$, provided, for each $r$, (1) holds, uniformly for $1 \leqslant d_{1}, \ldots, d_{r} \leqslant h$, with $d_{1}, \ldots, d_{r}$ distinct and $\mathscr{S}_{\mathrm{d}} \neq 0$. 
Our argument for (2) goes through a computation of the moments of $\pi(n+h)-\pi(n)$, and depends on the fact that, for each $r, \mathscr{S}_{\mathrm{d}}$ averages to 1 over cubes:

$$
\sum_{\substack{1 \leqslant d_{1}, \ldots, d_{r} \leqslant h \\ \text { distinct }}} \mathscr{S}_{\mathrm{d}} \sim h^{r} \quad(h \rightarrow \infty) .
$$

For $r=2$, a smoothed variant of this was used by Hardy and Littlewood to refute earlier asymptotic Goldbach conjectures. A simple proof of (3) for $r=2$, starting with the singular series representation for $\mathscr{S}_{\mathrm{d}}$, was given by Bombieri and Davenport in [1]. Our proof of (3) starts with the product definition of $\mathscr{S}_{\mathrm{d}}$, and is closer to an argument of Hooley in [5].

Using Selberg's sieve, Klimov [7] obtained for each $r$ the upper bound $\dagger$

$$
\pi_{\mathrm{d}}(N) \lesssim 2 r ! \mathscr{S}_{\mathrm{d}} \frac{N}{\log ^{r} N}
$$

for $N \rightarrow \infty$, uniformly for $\mathbf{d}$ in small cubes. For this, see Halberstam and Richert [2], Theorem 5.7. Using (4) instead of (1), we get upper bounds for the $k$ th moments of $\pi(n+h)-\pi(n)$ for $n \leqslant N$, as Bombieri and Davenport did for $k=2$. For large $k$, these bounds give

THEOREM 2. For positive constants $\mu \geqslant \lambda \geqslant 1$, the number of $n \leqslant N$ for which $\pi(n+\lambda \log N)-\pi(n)>\mu$ is $\lesssim N e^{-C \mu / \lambda}$, where $C$ is an absolute constant.

1. Reduction to (3). For each positive integer $k$,

$$
\begin{aligned}
\sum_{n \leqslant N}(\pi(n+h)-\pi(n))^{k} & =\sum_{n \leqslant N} \sum_{n<p_{1}, \ldots, p_{k} \leqslant n+h} 1 \\
& =\sum_{r=1}^{k} \sigma(k, r) \sum \pi_{d_{1}, \ldots, d_{r}}(N),
\end{aligned}
$$

where the inner sum is over all $r$-tuples $d_{1}, \ldots, d_{r}$ satisfying $1 \leqslant d_{1}<\ldots<d_{r} \leqslant h$, and $\sigma(k, r)$ is the number of maps from the set $\{1, \ldots, k\}$ onto $\{1, \ldots, r\}$. For the $\mathbf{d}$ with $\mathscr{S}_{\mathrm{d}} \neq 0$, we use (1); for the others, $d_{1}, \ldots, d_{r}$ occupy all residue classes modulo some prime, so $\pi_{\mathrm{d}}(N) \leqslant r$. Using (3), it follows that

$$
\sum \pi_{d_{1}, \ldots, d_{r}}(N) \sim \frac{h^{r}}{r !} \frac{N}{\log ^{r} N},
$$

and hence

$$
\frac{1}{N} \sum_{n=1}^{N}(\pi(n+h)-\pi(n))^{k} \rightarrow m_{k}(\lambda),
$$

with

$$
m_{k}(\lambda)=\sum_{r=1}^{k} \sigma(k, r) \frac{\lambda^{r}}{r !}
$$

In $\S 3$, it is shown that $m_{k}(\lambda)$ is the $k$ th moment of the Poisson distribution with

$\uparrow$ The notation $F \lesssim G$ stands for $\lim F / G \leqslant 1$. 
parameter $\lambda$, and that the corresponding moment generating function is entire. The result (2) now follows from general theorems on moments [6, Chapter 4].

Putting $h=\lambda \log N$, and using (4) instead of (1), we get

$$
\sum \pi_{d_{1}, \ldots, d_{r}}(N) \lesssim(2 \lambda)^{r} N,
$$

from which it follows that

$$
\begin{aligned}
\frac{1}{N} \sum_{n=1}^{N}(\pi(n+h)-\pi(n))^{k} & \lessgtr \sum_{r=1}^{k} \sigma(k, r)(2 \lambda)^{r} \\
& \leqslant k(2 \lambda k)^{k} .
\end{aligned}
$$

Hence the proportion of $n \leqslant N$ for which $\pi(n+h)-\pi(n) \geqslant \mu$ is $\leqslant k(2 k \lambda / \mu)^{k}$. If $\mu / \lambda \geqslant 4$, we choose $k=\left[\frac{1}{4}(\mu / \lambda)\right]$. Then $k \geqslant \frac{1}{8}(\mu / \lambda)$, so the proportion is

$$
\lesssim k 2^{-k} \leqslant e^{-c_{\mu / \lambda}} \text {. }
$$

If $\mu / \lambda<4$, the result is trivial.

2. Proof of (3). Let

$$
D_{\mathrm{d}}=\prod_{i<j}\left(d_{i}-d_{j}\right)
$$

Then $1 \leqslant v_{\mathrm{d}}(p) \leqslant r$, with equality at the right, unless $p \mid D_{\mathrm{d}}$. The $p$ th factor in $\mathscr{S}_{\mathrm{d}}$ is

$$
1+\frac{p^{r}-v_{\mathrm{d}}(p) p^{r-1}-(p-1)^{r}}{(p-1)^{r}}=1+a\left(p, v_{\mathrm{d}}(p)\right)
$$

where

$$
a(p, v) \ll, \begin{cases}(p-1)^{-2}, & v=r ; \\ (p-1)^{-1}, & v<r .\end{cases}
$$

It follows that the product for $\mathscr{S}_{\mathrm{d}}$ converges, Defining $a_{\mathrm{d}}(q)$ for squarefree $q$ by

$$
a_{\mathrm{d}}(q)=\prod_{p \mid q} a\left(p, v_{\mathrm{d}}(p)\right)
$$

we get an absolutely convergent series expansion

$$
\mathscr{P}_{\mathrm{d}}=\sum_{\mathbf{q}} a_{\mathrm{d}}(q)
$$

where the sum is over squarefree $q$.

We need an estimate for the remainder in (8) which is uniform for $\mathbf{d}$ in the $h$-cube. It follows from the bounds on $a(p, v)$ that

$$
\sum_{q>x}\left|a_{\mathrm{d}}(q)\right| \leqslant \sum_{q>x} \frac{\mu^{2}(q) C^{\omega(q)}}{\phi^{2}(q)} \phi((q, D)),
$$

where $\omega(q)$ is the number of prime factors of $q$, and $C$ is a positive constant depending only on $r$. Putting $q=d e$ with $d \mid D$ and $(e, D)=1$, this is

$$
\begin{aligned}
\sum_{d T_{b}} \frac{\mu^{2}(d) C^{\omega(d)}}{\phi(d)} \sum_{\substack{e>x / d \\
(e, D)=1}} \frac{\mu^{2}(e) C^{\omega(e)}}{\phi^{2}(e)} & \ll \sum_{d \mid D} \frac{\mu^{2}(d) C^{\omega(d)}}{\phi(d)} \frac{d}{x} \log ^{B} x \\
& \ll(x h)^{\ell} / x
\end{aligned}
$$


with a constant depending only on $r$ and $\varepsilon$. It follows that

$$
\sum_{\substack{d_{1} \\ \text { distinet }}} \mathscr{S}_{\mathrm{d}}=\sum_{q \leqslant x} \sum_{\substack{d_{r} \leqslant h \\ d_{1}, \ldots d_{r} \leqslant h \\ \text { distinct }}} a_{\mathrm{d}}(q)+O\left(h^{r}\left((x h)^{\varepsilon} / x\right)\right),
$$

with a constant depending only on $r$ and $\varepsilon$.

The inner sum in (9) is

$$
\sum_{v} \prod_{p \mid q} a(p, v(p))\left\{\Sigma^{\prime} 1+O\left(h^{r-1}\right)\right\}
$$

where $\sum^{\prime} 1$ stands for the number of $r$-tuples of not necessarily distinct integers $d_{1}, \ldots, d_{r}$ with $1 \leqslant d_{1}, \ldots, d_{r} \leqslant h$ which, for each prime $p \mid q$, occupy exactly $v(p)$ residue classes $\bmod p$; the outer sum is over all "vectors" $=(\ldots, v(p), \ldots)_{p \mid q}$ with components satisfying $1 \leqslant v(p) \leqslant p$. A simple lattice point argument using the Chinese remainder theorem gives, for $q \leqslant h$,

$$
\Sigma^{\prime} 1=\left\{\left(\frac{h}{q}\right)^{r}+o\left(\frac{h}{q}\right)^{r-1}\right\} \prod_{p \mid q}\left(\begin{array}{c}
p \\
v(p)
\end{array}\right) \sigma(r, v(p))
$$

the product representing the number of ways of choosing the residue classes of $d_{1}, \ldots, d_{r} \bmod q$ subject to the congruence restrictions in $\Sigma^{\prime}$.

Thus the inner sum in (9) is

$$
\left(\frac{h}{q}\right)^{r} A(q)+O\left(\left(\frac{h}{q}\right)^{r-1} B(q)\right)+O\left(h^{r-1} C(q)\right)
$$

with

$$
\begin{aligned}
& A(q)=\sum_{v} \prod_{p \mid q} a(p, v(p))\left(\begin{array}{c}
p \\
v(p)
\end{array}\right) \sigma(r, v(p)), \\
& B(q)=\sum_{v} \prod_{p \mid q}|a(p, v(p))|\left(\begin{array}{c}
p \\
v(p)
\end{array}\right) \sigma(r, v(p)), \\
& C(q)=\sum_{v} \prod_{p \mid q}|a(p, v(p))| .
\end{aligned}
$$

We have

$$
\begin{aligned}
& A(q)=\prod_{p \mid q}\left\{\sum_{v=1}^{p} a(p, v)\left(\begin{array}{l}
p \\
v
\end{array}\right) \sigma(r, v)\right\}, \\
& B(q)=\prod_{p \mid q}\left\{\sum_{v=1}^{p}|a(p, v)|\left(\begin{array}{l}
p \\
v
\end{array}\right) \sigma(r, v)\right\}, \\
& C(q)=\prod_{p \mid q}\left\{\sum_{v=1}^{p}|a(p, v)|\right\} .
\end{aligned}
$$

We show first that $A(q)=0$ for $q>1$. Using (6), the $p$ th factor in $A(q)$ is

$$
(p-1)^{-r}\left\{\left(p^{r}-(p-1)^{r}\right) \sum_{v=1}^{p}\left(\begin{array}{l}
p \\
v
\end{array}\right) \sigma(r, v)-p^{r-1} \sum_{v=1}^{p} v\left(\begin{array}{l}
p \\
v
\end{array}\right) \sigma(r, v)\right\} \text {. }
$$


By formulae (i) and (ii) of $\S 3$, the two sums here are $p^{r}$ and $p^{r+1}-(p-1)^{r} p$ respectively, and the factor vanishes.

Using the bounds (7) for $a(p, v)$, we may estimate $B(q)$ and $C(q)$. By (i) of $\S 3$, the $p$ th factor in $B(q)$ is $\ll p^{r} /(p-1)$, so

$$
B(q) \leqslant C^{\omega(q)} \frac{q^{r}}{\phi(q)} .
$$

More simply, the $p$ th factor in $C(q)$ is $\ll p /(p-1)$, so

$$
C(q) \leqslant C^{\omega(q)} \frac{q}{\phi(q)} .
$$

Returning to (9) and (10), it follows that (9) is $h^{r}$ plus a remainder term which is

$$
\begin{aligned}
& \ll h^{r-1} \sum_{q \leqslant x} C^{\omega(q)} \frac{q}{\phi(q)}+h^{r}(x h)^{\varepsilon} / x \\
& \ll h^{r-1} x^{1+\varepsilon}+h^{r}(h x)^{\varepsilon} / x \\
& \ll h^{r-\frac{1}{2}+\varepsilon},
\end{aligned}
$$

choosing $x=h^{\frac{1}{2}}$. Since $x \leqslant h$, the conditions $q \leqslant h$, assumed earlier, are satisfied.

3. Combinatorial identities. We prove here the standard identities for the "Stirling numbers of the second kind" $\sigma(k, r) / r$ ! which have been used above. These are
(i) $\sum_{\nu=1}^{p}\left(\begin{array}{l}p \\ v\end{array}\right) \sigma(r, v)=p^{r}$,
(ii) $\sum_{v=1}^{p} v\left(\begin{array}{l}p \\ v\end{array}\right) \sigma(r, v)=p^{r+1}-(p-1)^{r} p$
(iii) $\sum_{v=1}^{r} \sigma(r, v) \frac{\lambda^{v}}{v !}=\sum_{p=0}^{\infty} p^{r} \frac{e^{-\lambda} \lambda^{p}}{p !}$,
(iv) $\sum_{r=0}^{\infty} \frac{m_{r}(\lambda) z^{r}}{r !}=e^{-\lambda} e^{\lambda e^{z}}$;

the last two identities show that $m_{r}(\lambda)$, the left side of (iii), is the rth moment of the Poisson distribution with parameter $\lambda$, and that the corresponding moment generating function (iv) is entire.

To prove (i), classify the maps from $\{1, \ldots, r\}$ to $\{1, \ldots, p\}$ by the size of the image. There are $\left(\begin{array}{l}p \\ v\end{array}\right)$ subsets of size $v$ in $\{1, \ldots, p\}$; for each such subset, the number of maps with this image is $\sigma(r, v)$. To prove (ii), write

$$
\left(\begin{array}{l}
p \\
v
\end{array}\right)=p\left(\begin{array}{c}
p-1 \\
v-1
\end{array}\right)=p\left(\begin{array}{l}
p \\
y
\end{array}\right)-p\left(\begin{array}{c}
p-1 \\
v
\end{array}\right)
$$


and use (i). To prove (iii), multiply (i) by $\lambda^{p} / p$ ! and sum over $p$ :

$$
\sum_{\nu=1}^{r} \sigma(r, v) \sum_{p=0}^{\infty}\left(\begin{array}{l}
p \\
v
\end{array}\right) \frac{\lambda^{p}}{p !}=\sum_{p=0}^{\infty} p^{r} \frac{\lambda^{p}}{p !} .
$$

From this and

$$
\sum_{p=0}^{\infty}\left(\begin{array}{l}
p \\
v
\end{array}\right) \frac{\lambda^{p}}{p !}=\frac{\lambda^{v}}{v !} e^{\lambda}
$$

the identity (iii) follows. To prove (iv), multiply (iii) by $z^{r} / r$ ! and sum over $r$.

\section{References}

1. E. Bombieri and H. Davenport. "Small differences between prime numbers", Proc. Roy. Soc. Ser. A. 293 (1966), 1-18.

2. H. Halberstam and E. Richert. Sieve Methods (Academic Press, 1974).

3. G. H. Hardy and J. E. Littlewood. "Some problems of 'Partitio Numerorum': III. On the expression of a number as a sum of primes ", Acta Mathematica, 44 (1922), 1-70.

4. C. Hooley. "On the difference between consecutive numbers prime to $n$. II", Publ. Math. Debrecen, 12 (1965), 39-49.

5. C. Hooley. "On the intervals between consecutive terms of sequences", Proc. Symp. Pure Math., 24 (1973), 129-140.

6. M. G. Kendall and A. Stuart. The Advanced Theory of Statistics, Vol. I (Griffin, 1958).

7. N. I. Klimov. "Combination of elementary and analytic methods in the theory of numbers", Uspehi Mat. Nauk, 13 (1958), no. 3 (81), 145-164.

8. A. F.Lavrik. "On the theory of distribution of primes, based on I. M. Vinogradov's method of trigonometrical sums ", Trudy Mat. Inst. Steklov., 64 (1961), 90-125.

Mathematics Department

Columbia University

New York, New York 10027 (U.S.A.).
10H15: NUMBER THEORY; Multiplicative Theory; Distribution of primes.

Received on the 10th of October, 1975. 